International Journal of Nanoscience

Vol. 8, No. 3 (2009) 319-322

(c) World Scientific Publishing Company

\title{
FABRICATION AND EVALUATION OF HUMAN SERUM ALBUMIN (HSA) NANOPARTICLES FOR DRUG DELIVERY APPLICATION
}

\author{
R. MEHRAVAR ${ }^{*, \ddagger}$, M. JAHANSHAHI ${ }^{\dagger, \S}$ and N. SAGHATOLESLAMI*, \\ *Faculty of Chemical Engineering \\ Ferdowsi University of Mashhad, Mashhad, Iran \\ ${ }^{\dagger}$ Nanobiotechnology, Research Center \\ Babol University of Technology \\ Babol, P. O. Box 484, Iran \\ ${ }^{\ddagger}$ rabi_mehravar@yahoo.com \\ \$mjahan@nit.ac.ir \\ §mmohse@yahoo.com \\ গslami@um.ac.ir
}

\begin{abstract}
Human Serum Albumin (HSA) nanoparticles represent promising drug carrier systems. Particle size is a crucial parameter in particular for the in vivo behavior of nanoparticles after intravenous injection. The object of present study was to characterize the desolvation process of HSA for preparation of nanoparticles. Two process parameters were examined to achieve a suitable size of nanoparticles such as the $\mathrm{pH}$ value and the amount of glutaraldehyde concentration (\%).The smallest size of nanoparticles achieved was $91 \mathrm{~nm}$ and the largest size was $388 \mathrm{~nm}$ which is suitable for drug delivery. The $\mathrm{pH}$ value of the HSA solution prior to the desolvation procedure was identified as the major factor determining particle size and the amount of crosslinker showed that it has less effect on produced nanoparticle size. The nanoparticle sample was purified by five cycles' centrifugation $(20000 \times \mathrm{g}, 8 \mathrm{~min})$ and redispersion of the pellet to the original volume in $10 \mathrm{mM} \mathrm{NaCl}$ at $\mathrm{pH}$ values of $7.5-9$, respectively, and then analyzed by particle size analyzer (PCS).
\end{abstract}

Keywords: Human serum albumin; nanoparticles; drug delivery; desolvation method.

\section{Introduction}

Although, the drug delivery system (DDS) concept is not new, great progress has recently been made in the treatment of a variety of diseases. Targeting delivery of drugs to the diseased lesions is one of the most important aspects of DDS. To convey a sufficient dose of drug to the lesion, suitable carriers of drugs are needed. Nano- and microparticle carrier have important potential application for the administration of therapeutic molecules. The controlled DDSs offer numerous advantages compared to conventional dosage forms, which include

${ }^{\dagger}$ Corresponding author. 
improved efficiency, reduced toxicity, and improved patient compliance and convenience. Such systems often use macromolecules as carriers for the drugs. ${ }^{1}$

Nanoparticles are already in use in several areas of drug delivery and cosmetics. Usually less than $100 \mathrm{~nm}$, they are made by forming nanocrystals or drug-polymer complexes or by creating nanoscale shells (such as liposome) that entrap drug molecules. Nanoparticles have unusual properties that can be exploited to improve drug delivery. Because of their fine size, they are often taken up by cells where larger particles would be excluded or cleared from the body. Small molecules, peptides, proteins, and nucleic acids can be loaded into nanoparticles that are nonrecognized by the immune system and that can be targeted to particular tissue types. ${ }^{2}$

The major advantage of colloidal drug carrier systems is the possibility of drug targeting by a modified body distribution "see Ref. 3" as well as the enhancement of the cellular uptake. ${ }^{4}$

Among these colloidal systems, protein-based nanoparticles play an important role. The benefits of protein nanoparticles are: nontoxicity, stability for long duration, nonantigen, and also possesses biodegradability. ${ }^{5,6}$

The body distribution of colloidal DDSs is mainly influenced by two physicochemical properties, particle size and surface characteristics. ${ }^{7}$ Basically, three different methods for preparation of such nanoparticles (protein nanoparticles) have been described, based on emulsion formation, desolvation, and coacervation. The disadvantage of the emulsion methods for particle preparation is the need for applying organic solvents for the removal both of the oily residues of the preparation process and of surfactants required for emulsion stabilization. Therefore, as an alternative method for the preparation of nanoparticles as desolvation process derived from coacervation method of microencapsulation was developed.

Albumin is an alternative macromolecular carrier and widely used to prepare microspheres and microcapsules, due to its availability in pure form and its biodegradability, nontoxicity, and nonimmunogenicity. A number of studies have shown that albumin accumulates in solid tumors making it a potential macromolecular carrier for the site-directed delivery of antitumor drugs. ${ }^{1}$

Among these, human serum albumin (HSA) is a promising material and was used in a multitude of studies for particle preparation. ${ }^{8}$ HSA (molecular weight of $65 \mathrm{kDa}$ ) belongs to a multigene family of proteins "see Ref. 9" and is the major soluble protein of the circulating system with a blood concentration about $50 \mathrm{mg} \cdot \mathrm{ml}^{-1}$. HSA consists of 585 amino acids containing 35 cysteine residues which build 17 disulfide bridges.

The object of present study was to evaluate the desolvation of HSA for preparation of nanoparticles. Further investigations were focused on the influence of various $\mathrm{pH}$ values and the amount of glutaraldehyde concentration (\%) on the size of nanoparticles.

\section{Materials and Methods}

\subsection{Materials}

HSA (fraction V, purity 96-99\%) and glutaraldehyde solution were commercially supplied by Sigma. Ethanol and other reagents were purchased from Merck.

\subsection{Preparation of HSA nanoparticles}

HSA nanoparticles were prepared by a desolvation technique as described previously. ${ }^{10}$ In principle, between 50 and $200 \mathrm{mg}$ HSA was dissolved in $2 \mathrm{ml}$ of purified water or $10 \mathrm{mM} \mathrm{NaCl}$ solution, then titrated to $\mathrm{pH}$ 7.5-9 and under constant stirring desolvation of HSA solution was achieved by dropwise addition of ethanol. After the desolvation process, $8 \%$ glutaraldehyde in water (between 0.235 and $1.175 \mu \mathrm{l} / \mathrm{mg}$ HSA) was added to induce particle crosslinking. Crosslinking process was performed under stirring of the suspension over a time period of $24 \mathrm{~h}$. The resulting nanoparticles were purified by five cycles of centrifugation $(20000 \times \mathrm{g}, 8 \mathrm{~min})$ and redispersion of the pellet to the original volume in $10 \mathrm{mM} \mathrm{NaCl}$ at $\mathrm{pH}$ values of $7.5-9$, respectively.

\subsection{Determination of nanoparticles size and distribution}

The size distribution of the prepared HSA nanoparticle was analyzed by photon correlation spectroscopy (PCS). The sample analyzed in the PCS device should consist of well-dispersed particles in liquid medium. In such condition, the particles are in constant random motion, referred to as Brownian motion and PCS measures the speed of this motion by passing a laser. PCS determines the average particle size and polydispersity Index (PI) which is a 
range of measurement of the particle size within measured samples. The accurate measurement of particle size must be blow 0.7 (70\%).

\section{Results and Discussion}

\subsection{The effect of different parameters on nanoparticle size}

The impact of $\mathrm{pH}$ value and the amount of glutaraldehyde concentration (\%) on the nanoparticle size was studied.

The $\mathrm{pH}$ value of the HSA solution prior to ethanol addition influenced the resulting particle size. After investigation of the effect of $\mathrm{pH}$ on nanoparticle size, it was found that with increasing $\mathrm{pH}$ value of HSA solution particle size were reduced, apparently due to an increased ionization of the HSA which leads to repulsion of the HSA molecules and aggregates during particle formation. This result is in good agreement with the earlier work of Lin and his coworkers. ${ }^{11}$

The effect of $\mathrm{pH}$ on nanoparticle size was conducted and shown in Table 1.

After the desolvation procedure the nanoparticles were crosslinked, per milligram HSA, with $0.235,0.588$, or $1.175 \mu \mathrm{l}$ of an aqueous $8 \%$ glutaraldehyde solution. These glutaraldehyde concentrations equal 40,100, and $200 \%$ of the calculated amount necessary for the quantitative crosslinking of the $59 \varepsilon$-amino groups of lysine in the HSA molecules of the particle matrix. ${ }^{12}$ Whereas no influence of the crosslinking conditions on the resulting particle size was observed, the glutaraldehyde crosslinking of the particle matrix tends to result in a decrease in the isoelectric point (pI) of the nanoparticles. The reduction in the pI value could be expected for a covalent reaction involving lysine side chains of a protein.

According to Table 2, no influence of the crosslinking conditions on the resulting particle size

Table 1. Effect of $\mathrm{pH}$ on nanoparticle size and polydispersity.

\begin{tabular}{lrc}
\hline $\mathrm{pH}$ & Particle size $(\mathrm{nm})$ & Polydispersity \\
\hline 7.5 & 388 & 0.171 \\
8 & 93 & 0.110 \\
8.5 & 91 & 0.105 \\
9 & 91 & 0.105 \\
\hline
\end{tabular}

Table 2. Effect of glutaraldehyde concentration (\%) on nanoparticle size and polydispersity.

\begin{tabular}{ccc}
\hline $\begin{array}{l}\text { Glutaraldehyde } \\
\text { concentration (\%) }\end{array}$ & Particle size (nm) & Polydispersity \\
\hline 40 & 111 & 0.124 \\
100 & 99 & 0.120 \\
200 & 91 & 0.105 \\
\hline
\end{tabular}

observed. A similar result was declared on fabrication of protein nanoparticles using bovine serum albumin (BSA). ${ }^{6}$

\section{Conclusions}

The present study shows that HSA nanoparticles can be prepared with desolvation process. The nanoparticles size fabricated from HSA was influenced by several process variable including $\mathrm{pH}$ value, HSA concentration, the amount of glutaraldehyde concentration (\%), ratio of desolvating agent/ HSA, rate of addition of the desolvating agent, and agitation speed. In this study, the effect of $\mathrm{pH}$ value and the amount of glutaraldehyde concentration (\%) on the particle size was investigated. In predetermined conditions (protein concentration $100 \mathrm{mg} \cdot \mathrm{ml}^{-1}$ ), the particle was prepared at $25^{\circ} \mathrm{C}$, $\mathrm{pH}=9$, and agitation speed $550 \mathrm{rpm}$. The minimum size of particle fabricated was $91 \mathrm{~nm}$.

Optimization of this fabrication method for protein nanoparticles as DDSs will be the subject of next publication.

\section{Acknowledgments}

The authors would like to gratefully acknowledge members of the Nanobiotechnology Research Laboratory of Babol University of Technology, and Chemical Engineering Research Laboratory of Ferdowsi University of Mashhad in Iran.

\section{References}

1. N. V. Majeti and R. Kumar, J. Pharm. Pharmaceut. Sci. 3, 234 (2000).

2. D. Alavan, T. McGuire and R. Langer, Nature Biotechnol. 21, 1184 (2003).

3. J. Kreuter, Pharm. Acta Helv. 58, 217 (1983).

4. V. Schäfer, H. Von Briesen, R. Andreesen, A. M. Steffan, C. Royer, S. Tröster, J. Kreuter and H. Rübsamen-Waigmann, Pharm. Res. 9, 541 (1992).

5. B. Muller, H. Levenberger and T. Kissel, Pharm. Res. 13, 32 (1996). 
6. M. Rahimnejad, M. Jahanshahi and G. Najafpour, Afr. J. Biotechnol. 5, 1918 (2006).

7. M. Jahanshahi, G. Najafpour and M. Rahimnejad, Afr. J. Biotechnol. 7, 362 (2008).

8. K. Michaelis, M. M. Hoffmann, S. Dreis, E. Herbert, R. N. Alyatdin, M. Michaelis, J. Kreuter and K. Langer, L. Pharm. Exp. Ther. 317, 1246 (2006).
9. X. M. He and D. C. Carte, Nature 358, 209 (1992).

10. J. J. Marty, R. C. Oppenheimer and P. Speiser, Pharm. Acta Helv. 53, 17 (1978).

11. W. Lin, A. G. Coombes, M. C. Davies, S. S. Davis and L. Illum, J. Drug Target 1, 237 (1993).

12. D. C. Carter and J. X. Ho, Adv. Protein Chem. 45, 153 (1994). 